\section{Autologous Stromal Vascular Fraction: A New Era of Personal Cell Therapy}

\author{
Elliot B Lander* and Mark H Berman \\ California Stem Cell Treatment Center, Cell Surgical Network Inc., California, \\ USA
}

\begin{abstract}
The fascinating story of Stromal Vascular Fraction (SVF) and its prominent role in autologous regenerative therapies (or Personal Cell Therapy - PCT) continues to evolve as scientific and regulatory issues develop. The primary objective of this special report will elucidate the history, composition and scientific analysis of SVF as an autologous biologic experiencing widespread clinical use. SVF has several distinct features that prevent it from ever being manufactured as a mass produced pharmaceutical and distributed for clinical use. Autologous SVF contains a unique mixture of 4 different types of autologous stem cells in an extremely rich cytokine milieu. It is intended to be used fresh and at point of care obviating its use in commercialization. It is personalized with the patient's own DNA, and it contains the patient's own biologic profile of possible measurable or unmeasurable communicable diseases. Other stem cell products being developed as drugs under research and development differ from autologous SVF as they generally contain doses of a single cell types that may or may not be autologous. Personal Cell Therapy using autologous biologic products should be protected from overbearing regulation with its use supported by clinical trials helping us to learn more about its regenerative potential. Laboratory investigations can then optimally help optimize areas that need further support.
\end{abstract}

Keywords: Hematopoietic stem cells; Mesenchymal stem cells; Stem cell therapy; Stromal vascular fraction

*Corresponding author: Elliot B Lander, California Stem Cell Treatment Center, Cell Surgical Network Inc., 72780 Country Club Drive \#301, Rancho Mirage, California, USA, Tel: +1 7603460145; Fax: +1 7607760041; E-mail: elliot@cellsurgicalnetwork.com

Citation: Lander EB, Berman MH (2018) Autologous Stromal Vascular Fraction: A New Era of Personal Cell Therapy. J Stem Cell Res Dev Ther 4: 011.

Received: November 16, 2018; Accepted: December 03, 2018; Published: December 19, 2018

Copyright: (c) 2018 Lander EB and Berman MH. This is an open-access article distributed under the terms of the Creative Commons Attribution License, which permits unrestricted use, distribution, and reproduction in any medium, provided the original author and source are credited.

\section{History of SVF}

Stromal Vascular Fraction (SVF) procured from adipose tissue lipoaspirate represents stromal tissue that contains a variety of different stem cells as well as other supporting cells and signaling molecules. This unique collection of cells act together to orchestrate and facilitate a number of biologic processes including: accelerating healing, decreasing inflammation, modulating the immune system, and a number of cytokine mediated local and systemic effects.

Mesenchymal cells derived from fat have apparently been studied for several decades but in 2001 Zuk PA et al., at the UCLA laboratory for regenerative bioengineering and repair formally described them when they determined that a population of stem cells could be isolated from lipoaspirate processed from human adipose tissue via liposuction [1]. The investigative team noted that these cells could be maintained in vitro for extended periods and that the cells appeared to be of mesenchymal origin. The following year they published a second paper continuing their research and showing isolation of a putative stem cell population from human adipose tissue containing stroma [2]. In 2002, Stafford KM et al., showed that adipose derived human stem cells can be induced to undergo neuronal differentiation and were ideal for tissue regeneration [3]. The cells appeared to have particular capability of forming cardiomyocytes and also appeared to be effective in mitigating autoimmune conditions. Froehlich $\mathrm{H}$ et al., at Mayo Clinic reported that adipose tissue is also an abundant source of endothelial cells including stem and progenitor cells [4]. These cells had excellent angiogenic properties both in vivo and in vitro and they apparently had the ability to rapidly improve re-endothelialization. Over the next few years, hundreds of publications have confirmed and described similar results demonstrating the presence and utility of mesenchymal stem cells found in adipose tissue.

Despite many elegant articles coming from laboratory studies, the scientific history of stromal vascular fraction is truly rooted in the cosmetic surgery world since the science was driven by a need to improve fat grafting. Although cosmetic and reconstructive fat transfers had significant functional and cosmetic advantages, limitations included unpredictable and often non-sustainable outcomes. The concept of cell assisted lipo-transfer to improve cosmetic outcomes developed when it became apparent that if you combine a free fat graft with stromal vascular fraction derived from fat, the stem cell rich environment stimulated by hypoxic transplant conditions appeared to improve the long-term viability of the transferred fat tissue [5]. Nevertheless, it became apparent during early clinical experience of using stromal vascular fraction that it could also be used for therapeutics and the utility extended far beyond cosmetics.

\section{Isolation of SVF}

There are multiple methods of preparing stromal vascular fraction many of which involve a laboratory procedure. Many methods of production are manual or fully automated. Our research group, Cell Surgical Network(CSN), uses a semi-manual "surgically closed" syringe system in order to harvest and process liposuction fat into SVF. 
Our CSN procurement system offers a surgical procedure that uses specially adapted syringes that can be centrifuged with their contents transferred from syringe to syringe using sterile adapters on the sterile field. The entire procedure performed in an operating area in the clinic requires no laboratory equipment such as hoods or pipettes. The occasional exposure of the syringe aperture to air is consistent with acceptable surgical standards. We use the concept of "surgically closed" to emphasize two important concepts. First, no human can be guaranteed to be free of some type of contamination (e.g. viral or prions) as we are not raised in sterile environments. Indeed, humans with known contaminants could never be used to make a mass produced drug product, yet their own cells would cause them no harm. Second, the process of liposuction is practically impossible to perform without exposure to ambient air. Surgery would have to be done in an approved "clean room" similar to pharmaceutical manufacturing requirements and this hasn't yet been found to be necessary according to current surgical operating accreditation standards. Acceptable surgical standards based upon a single operation on a single patient differ significantly from the Good Manufacturing Practices (GMP) standards established for mass produced pharmaceuticals. In surgery, billions of our patient's cells are routinely exposed to ambient air through incisions. With a mass produced pharmaceutical this contact with the environment needs to be avoided for obvious reasons - the manufacturer is making huge batches and wouldn't want to risk contamination that would ruin large quantities of drug products and it would be far more practical to have a validated clean system to avoid necessity of testing all of your final end products. A manufactured drug requires the end-product to be uniform in dose, strength and purity while autologous SVF could never be characterized that way. Every "dose" or deployment of SVF is individualized like a snowflake.

$\mathrm{CSN}$ as a clinical research organization has accumulated safety data on over 9500 personalized surgical procedures performed under IRB as off all 2018 without a single infection caused by adulterated processing of SVF. While SVF in humans is most commonly used clinically as an autologous product during a point of care procedure, notably, on rare occasions (e.g. special circumstances with a primary relative in which the patient cannot undergo the liposuction procedure) allogeneic donated SVF may also be used for cell therapy.

\section{Content of SVF}

Although there are a variety of methods for obtaining adipose derived stem cells, the combination of liposuction and collagens enzyme is particularly efficient. Together they not only separate cells from collagen but they also release cells from the vascular component of stromal vascular fraction and this likely provides the most variety and quality of stem cells. CSN contracted independent laboratories have performed flow cytometric immuno-phenotyping of multiple samples of autologous stromal vascular fraction demonstrating four distinct robust stem cell lines in varying ratios including mesenchymal stem cells, hematopoietic stem cells, endothelial progenitor cells, and pericyte (lying on top of blood vessels) progenitor cells [6], and a number of non-progenitor cells such as T-reg cells, macrophages, epithelial cells, and red and white blood cells as well as a rich soup of cytokines. Similar findings have been reported in previous studies of SVF composition [7] IFATS and ISCT reviewed the literature and published a position paper defining SVF as containing cells that are phenotypically identified by the following surface antigen markers: CD45-, CD 235a-, CD31-, CD34+, CD13+, CD73+, CD90+ CD105+ [8].
The cell markers help define international standards and reproducible parameters when evaluating the literature but much more important is to understand that SVF is not just adipose tissue but mesenchymal supporting tissue found in and around the adipose layer. See Michelek's description of SVF from his paper summarizing the Czech Republic experience with 1856 intra-articular SVF injections on 1128 patients.

In the vast majority of scientific publications only the term adipose tissue is used, but the true source of SVF cells is not the adipose part but only the stromal (i.e., loose connective tissue) part of the fat obtained typically by liposuction. Histologically, the fat lobules are surrounded by a loose connective tissue and the SVF cells reside in the loose connective tissue that also home capillaries and small vessels. Stroma is a broadly used term for the loose connective tissue that contains mesenchymal stem cells and other cells like fibroblasts, macrophages, adipocytes, mast cells and leukocytes. Synovia of articulated joints is also intimately associated with the loose connective tissue which is homologous to the loose connective tissue of the adipose tissue [9].

\section{Mechanism of Action of SVF}

The mechanism of action of mesenchymal stem cells found in SVF for mitigating damage and degeneration is in some ways still poorly understood. At first it was assumed that the cells must engraft and trans-differentiated in order to create new cells to effect repair but now we are starting to understand that this is not always the case. Stem cells are in constant communication through biologic signaling with the surrounding cell milieu and also in feedback loop signaling on their own cell membranes. This signaling system allows stem cells to induce healing and influence damaged target cells from proximity without actual engraftment in each case [10]. This cell to cell communication can be effected through cytokines or exosomes [11] which can contain RNA, mRNA, MicroRNA, DNA, or peptides. Stem repair cells in SVF like all stem cells are able to detect and interpret bio-signal codes from damaged or degenerated tissue resulting in homing and activation activity.

\section{Clinical Use of SVF}

Since all medical interventions ultimately accentuate and improve the patient's ability to defend against damage or disease (immune function) and to heal (repair or stem cell function) at a cellular level, the clinical applications of stromal vascular fraction are extensive. Stem cells help us heal from trauma, including the iatrogenic trauma of surgery. They can also help us heal from infections, autoimmune diseases, degenerative diseases and probably even age-related conditions. In fact, any assault on the body that harms cellular integrity may be mitigated by cell therapy.

The concept behind deployment of SVF is to amplify of our natural repair system by exogenously introducing increased numbers of autologous healing cells as a biologic "surge" in proximity to the damaged tissue. The SVF cells have always been present and capable of repair but not bio-accessible since they were locked in a sub-cutaneous collagen matrix. The subcutaneous collagen matrix locks them in place where they heal lacerations and respond to caloric challenge by making pre-adipocytes. They also have numerous endocrine functions and fat is now officially described as an endocrine organ as opposed to an inert tissue that stores fat as was once previously thought [12]. The ability to liberate these cells in the form of SVF and exploit 
their profound healing properties will ultimately expand our traditional sources of healing and synergize with any other medical treatment physicians and surgeons currently provide.

Considering the "cell factor" efforts have been underway to determine which stem cells are most effective for a particular condition. Despite many claims that certain types of stem cells are greatly superior to those from alternate sources, little proof exists that there are great differences between sources of non-engineered progenitor stem cells if you compare the cells on a functional basis. Any mammalian stem cells regardless whether the source is from bone marrow, neonatal tissues, or adipose likely have relatively similar differentiation and proliferative functional capabilities. All cells can probably function similarly to mitigate cellular damage although they may have slightly different antigenic cell markers. Several in vitro studies have been published supposedly showing that one type of stem cell is seemingly better than another type for certain purposes but many of these studies are industry biased and used to market a particular product. It remains unclear whether such cell source differences are accurate in vivo and clinically relevant. Further, most laboratory based studies on SVF have been done with fat obtained following traditional liposuction. Such studies often show sub-optimal adipose stem cell activity and may even suggest that adipose stem cells may be inferior to other biologic sources. Unfortunately, such cell samples are typically withdrawn using tumescent local anesthesia, most frequently, lidocaine, which is cytotoxic and the actual reason for the perceived poor cell function. This issue can be essentially eliminated by using a sub-dermal method of local anesthesia and rapid procurement to isolate the adipose tissue for production of SVF. Ultimately, the "cell factor" which distills down to how can we get large quantities of healthy stem cells, extract them and re-deploy them into target tissue in a timely, safe and sterile manner.

Having addressed the "cell factor," it is vital to underscore the importance of the "patient factor" to the clinical success of cell therapy. Clinically, based on our nearly 10,000 patient experiences, we've learned that the state of the injury is probably the most important determinant for success in treatment. This is the likelihood that the target tissue regenerative milieu will support the homing and activation of the repair cells. Under these circumstances, very extensive and very acute injuries giving off "loud damage signals" will likely be more successful with cell therapy than chronic indolent smoldering low-grade "quiescent" degenerative problems. Clinical efforts can be made to influence the "patient factor" to optimize cell therapy and one example is the addition of low intensity shock waves to SVF for the cell therapy treatment of erectile dysfunction (a relatively indolent non-inflammatory condition). The concept is to make the penile tissue signals "louder" in an iatrogenic attempt to enhance the homing and activation of exogenously supplied stem cells in the form of autologous SVF [13].

As far as autologous versus allogeneic sources of cells, it would seem prudent to use autologous cells if at all possible since they share the person's own DNA and if prepared in a sterile manner, there's no risk of transmissible disease from another source. As far as bone marrow versus adipose derived cells, a mini liposuction can be done essentially painlessly yielding higher quantities of stem cells. Furthermore, older and sicker patients tend to have suppressed bone marrow function. This issue may be less important in younger people with orthopedic injuries but in much older patients with systemic disease, the use of stem cells from depleted bone marrow become less compelling. Adipose stem cells aren't constantly replicating as are bone marrow cells. Adipose stem cells replicate under the pressure of increased caloric intake, under the influence of certain medications, such as steroids, or when signaled by a dying or injured adipose cell. This suggests that these cells are replicating a few dozen times during most people's lifetime, something rather insignificant for the life of a cell.

\section{Safety of SVF}

The safety record for autologous stem cell use has been quite excellent. Historic evidence of safety comes from the animal world where SVF has been extensively used as a regenerative therapy, particularly dogs and racehorses, with excellent safety and efficacy. Thousands of veterinary patients have received intra-articular autologous SVF to mitigate arthritis and orthopedic injuries successfully $[14,15]$.

Regarding human safety, SVF continues to have superb safety rates in clinical use. In one review of over 1000 clinical trials on stem cells, the conclusion was that there were no publications that present stem cells as harmful and that it is therefore nonessential to repeat clinical trials for non-expanded autologous stem cell therapies [16]. Arguably, there is no known "drug" as safe as your own personal SVF or stem cell products which can never be considered a "drug" product.

Interestingly, human experience with isolated SVF has been described in the last 17 years but technically the clinical use of SVF goes back decades. We now know that every liposuction procedure mechanically dissociates the adipose cells so that every liposuction and cosmetic lipo-transfer procedure performed over the last 30 years has freely circulating SVF cells. This dilute SVF product has been serendipitously used in tens of thousands of procedures. Thus, this indirect clinical evidence of safety is actually quite compelling and it is important to remember that autologous SVF and SVF-like biological are not brand new and not risky to patients.

Our group has published safety data on over 1698 deployments in 1524 patients [17]. This included regional and intravenous deployment of SVF. We have learned that intravenous infusions are safe with only one case of pulmonary embolism (in a wheelchair bound patient not directly related to cell therapy) after over 9500 procedures. We have also determined that IV infusions improve clinical outcomes since the cells "home" naturally to all parts of the body that are damaged based on cytokine and damage signal beacons. This is a natural targeting system and makes the cells "smart." When you combine intravenous systemic injection with physician directed regional deployments, such as intra-articular or into an organ, efficacy outcomes appear to be optimized. We recommend that SVF be prepared in such a way that it is filtered through $100 \mu \mathrm{m}$ filters to make intravenous infusion safe.

\section{Clinical Trials of SVF}

There have been a large number of clinical trials using SVF for a wide variety of conditions including but not limited to neurologic diseases, wound care, cardiac problems, autoimmune conditions, COPD, and orthopedic conditions [18-27]. The fundamental issue of researching SVF is that most of the trials have been empiric studies and only few have been done with a placebo arm. This is a common criticism of the SVF industry but it is always the case with surgical procedures. It is extremely difficult to perform sham surgeries and 
give patients a placebo product after they went through a liposuction procedure. It is important to remember that the entire field of surgery has advanced through empiric methods. There is no double blind placebo study showing that rhinoplasty works or that appendectomy is better than no appendectomy. Although adhering to surgical principles rather than drug investigation protocols is important for the evaluation of SVF there is still a role for placebo studies moving forward and our research group has applied for FDA approval to do a 40 patient placebo knee study for safety and efficacy.

The other fundamental issue regarding the study of SVF is that funding for well performed trials has been lacking. Although autologous point of care procedures generate revenue for the physician performing the procedure, there is no scaled up pharmacologic business model for SVF that is attractive to a pharmaceutical company seeking to develop a new drug since SVF as an autologous unique biologic could never be patented and then bottled and commercialized for distribution. This lack of business interest has trickled down into a lack of grants and funding and a requirement for most clinicians doing SVF research to rely on patient funding models and occasionally on charity.

\section{Regulation of SVF}

There are currently significant regulatory issues surrounding the clinical production and use of SVF by physicians. Recent FDA draft guidance language recently classifies SVF as a "drug." If that remains unchallenged, SVF will have to be manufactured and approved in an FDA regulated manner as opposed to isolating it as a surgical procedure in an operating room where it comes under the domain of the surgeon to produce and deploy the cells for their patient's benefit. Should SVF definitions prevail, the stringent regulatory requirements to manufacture and license SVF would likely extinguish the technology overnight in the USA with repercussions around the world. Funding for SVF research would become even more scarce.

\section{Conclusion}

Despite regulatory and financial hurdles, the medical and intellectual future of personal cell therapy using autologous SVF remains optimistic. We have seen positive outcomes using SVF at point of care in mitigating a number of medical problems and diseases. One of the limitations of autologous point of care SVF is that certain conditions such as advanced neurodegenerative disorders and autoimmune disorders seem to require repeat treatments or higher cell counts than cannot be realistically obtained at a point of care procedure using SVF. This opens up the door for cryobanking and expanding SVF cells. Only one lineage of mesenchymal stem cells is produced in these expanded cultures but very high numbers can be achieved conveniently and banked for future uses. Industrialized production of one's own stem cells is potentially a powerful tool that could keep us living longer and better. In synergy with our in-vivo repair system, large numbers of banked cells can be exogenously deployed as a "surge" to mitigate many conditions associated with trauma, radiation, surgery, violence, infection, behavioral and lifestyle issues (e.g. tobacco abuse), work or play overutilization injuries, genetic and epigenetic conditions (both mental and physical), toxins, cancer, and even ageing. This hails the beginning of true personalized medical treatments.

\section{Funding}

Funding was provided by Cell Surgical Network.

\section{References}

1. Zuk PA, Zhu M, Mizuno H, Huang J, Futrell JW, et al. (2001) Multilineage cells from human adipose tissue: implications for cell-based therapies. Tissue Eng 7: 211-228.

2. Zuk PA, Zhu M, Ashjian P, De Ugarte DA, Huang JI, et al. (2002) Human adipose tissue is a source of multipotent stem cells. Mol Biol Cell 13: 4279-4295.

3. Safford KM, Hicok KC, Safford SD, Halvorsen YD, Wilkison WO, et al. (2002) Neurogenic differentiation of murine and human adipose-derived stromal cells. Biochem Biophys Res Commun 294: 371-379.

4. Froelich H, Gulati R, Boilson B, Witt T, Harbuzariu A, et al. (2009) Carotid repair using autologous adipose-derived endothelial cells. Stroke 40: 1886-1891.

5. Tan SS, Ng ZY, Zhan W, Rozen W (2016) Role of Adipose-derived Stem Cells in Fat Grafting and Reconstructive Surgery. J Cutan Aesthet Surg 9: 152-156.

6. Kilinc MO, Santidrian A, Minev I, Toth R, Draganov D, et al. (2018) The ratio of ADSCs to HSC-progenitors in adipose tissue derived SVF may provide the key to predict the outcome of stem-call therapy. Clin Trans Med 7: 5.

7. Yoshimura K, Shigeura T, Matsumoto D, Sato T, Takaki Y, et al. (2006) Characterization of freshly isolated and cultured cells derived from the fatty and fluid portions of liposuction aspirates. J Cell Physiol 208: 64-76.

8. Bourin P, Bunnell B, Casteilla L, Dominici M, Katz AJ, et al. (2013) Stromal cells from the adipose tissue-derived stromal vascular fraction and culture expanded adipose tissue-derived stromal/stem cells: a joint statement of the International Federation of Adipose Therapeutics (IFATS) and Science and the International Society for Cellular Therapy (ISCT). Cytotherapy 15: 641-648.

9. Michalek J, Moster R, Lukac L, Proefrock K, Petrasovic M, et al. (2017) Stromal vascular fraction cells of adipose and connective tissue in people with osteoarthritis: A case control prospective multi-centric non-randomized study. Glob Surg 3: 1-9.

10. Gnecchi M, Danieli P, Malpasso G, Ciuffreda MC, et al. (2016) Paracrine Mechanisms of Mesenchymal Stem Cells in Tissue Repair. Methods Mol Biol 1416: 123-146.

11. Lai RC, Yeo RW, Lim SK (2015) Mesenchymal stem cell exosomes. Semin Cell Dev Biol 40: 82-88.

12. Coelho M, Oliveira T, Fernandes R (2013) Biochemistry of adipose tissue: an endocrine organ. Arch Med Sci 9: 191-200.

13. Lander EB, Berman MH (2018) Autologous Stromal Vascular Fraction Containing Stem Cells Combined with Low Intensity Shock Wave Treatment of Human Erectile Dysfunction. J Stem Cell Res Ther 8: 9.

14. Astor DE, Hoelzler MG, Harman R, Bastian RP (2013) Patient factors influencing the concentration of stromal vascular fraction (SVF) for adipose-derived stromal cell (ASC) therapy in dogs. Can J Vet Res 77: 177182.

15. Marx C, Silveira MD, Beyer Nardi N (2015) Adipose-derived stem cells in veterinary medicine: characterization and therapeutic applications. Stem Cells Dev 24: 803-813.

16. Van Pham P (2016) Clinical trials for stem cell transplantation: when are they needed? Stem Cell Res Ther 7: 65.

17. Berman M, Lander E (2017) A Prospective Safety Study of Autologous Adipose-Derived Stromal Vascular Fraction using a specialized Surgical Processing System. The American Journal of Cosmetic Surgery 34: 129142. 
18. Atalay S, Coruh A, Deniz K (2014) Stromal vascular fraction improves deep partial thickness burn wound healing. Burns 40: 1375-1383.

19. Rajashekhar G, Ramadan A, Abburi C, Callaghan B, Traktuev DO, et al (2014) Regenerative therapeutic potential of adipose stromal cells in early stage diabetic retinopathy. PloS One 9: 84671.

20. Premaratne GU, Ma LP, Fujita M, Lin X, Bollano E, et al. (2011) Stromal vascular fraction transplantation as an alternative therapy for ischemic heart failure: anti-inflammatory role. J Cardiothorac Surg 6: 43.

21. Mohammadi R, Sanaei N, Ahsan S, Rostami H, Abbasipour-Dalivand S, et al. (2014) Repair of nerve defect with chitosan graft supplemented by uncultured characterized stromal vascular fraction in streptozotocin induced diabetic rats. Int J Surg 12: 33-40.

22. Semon JA, Zhang X, Pandey AC, Alandete SM, Maness C, et al. (2013) Administration of murine stromal vascular fraction ameliorates chronic experimental autoimmune encephalomyelitis. Stem Cells Transl Med 2: 789-796.

23. Han SK, Kim HR, Kim WK (2010) The treatment of diabetic foot ulcers with uncultured, processed lipoaspirate cells: a pilot study. Wound Repair Regen 18: 342-348.
24. Rigotti G, Marchi A, Galiè M, Baroni G, Benati D, et al. (2007) Clinical treatment of radiotherapy tissue damage by lipoaspirate transplant: a healing process mediated by adipose-derived adult stem cells. Plast Reconstr Surg 119: 1409-1422.

25. Dalal J, Gandy K, Domen J (2012) Role of mesenchymal stem cell therapy in Crohn's disease. Pediatr Res 71: 445-451.

26. Mehrkens A, Saxer F, Güven S, Hoffmann W, Müller AM, et al. (2012) Intraoperative engineering of osteogenic grafts combining freshly harvested, human adipose-derived cells and physiological doses of bone morphogenetic protein-2. Eur Cell Mater 24: 308-319.

27. Jurgens WJ, Kroeze RJ, Zandieh-Doulabi B, van Dijk A, Renders GA, et al. (2013) One-step surgical procedure for the treatment of osteochondral defects with adipose-derived stem cells in a caprine knee defect: a pilot study. Biores Open Access 2: 315-325. 


\section{di}

Journal of Anesthesia \& Clinical Care

Journal of Addiction \& Addictive Disorders

Advances in Microbiology Research

Advances in Industrial Biotechnology

Journal of Agronomy \& Agricultural Science

Journal of AIDS Clinical Research \& STDs

Journal of Alcoholism, Drug Abuse \& Substance Dependence

Journal of Allergy Disorders \& Therapy

Journal of Alternative, Complementary \& Integrative Medicine

Journal of Alzheimer's \& Neurodegenerative Diseases

Journal of Angiology \& Vascular Surgery

Journal of Animal Research \& Veterinary Science

Archives of Zoological Studies

Archives of Urology

Journal of Atmospheric \& Earth-Sciences

Journal of Aquaculture \& Fisheries

Journal of Biotech Research \& Biochemistry

Journal of Brain \& Neuroscience Research

Journal of Cancer Biology \& Treatment

Journal of Cardiology: Study \& Research

Journal of Cell Biology \& Cell Metabolism

Journal of Clinical Dermatology \& Therapy

Journal of Clinical Immunology \& Immunotherapy

Journal of Clinical Studies \& Medical Case Reports

Journal of Community Medicine \& Public Health Care

Current Trends: Medical \& Biological Engineering

Journal of Cytology \& Tissue Biology

Journal of Dentistry: Oral Health \& Cosmesis

Journal of Diabetes \& Metabolic Disorders

Journal of Dairy Research \& Technology

Journal of Emergency Medicine Trauma \& Surgical Care

Journal of Environmental Science: Current Research

Journal of Food Science \& Nutrition

Journal of Forensic, Legal \& Investigative Sciences

Journal of Gastroenterology \& Hepatology Research
Journal of Gerontology \& Geriatric Medicine

Journal of Genetics \& Genomic Sciences

Journal of Hematology, Blood Transfusion \& Disorders

Journal of Human Endocrinology

Journal of Hospice \& Palliative Medical Care

Journal of Internal Medicine \& Primary Healthcare

Journal of Infectious \& Non Infectious Diseases

Journal of Light \& Laser: Current Trends

Journal of Modern Chemical Sciences

Journal of Medicine: Study \& Research

Journal of Nanotechnology: Nanomedicine \& Nanobiotechnology

Journal of Neonatology \& Clinical Pediatrics

Journal of Nephrology \& Renal Therapy

Journal of Non Invasive Vascular Investigation

Journal of Nuclear Medicine, Radiology \& Radiation Therapy

Journal of Obesity \& Weight Loss

Journal of Ophthalmology \& Clinical Research

Journal of Orthopedic Research \& Physiotherapy

Journal of Otolaryngology, Head \& Neck Surgery

Journal of Protein Research \& Bioinformatics

Journal of Pathology Clinical \& Medical Research

Journal of Pharmacology, Pharmaceutics \& Pharmacovigilance

Journal of Physical Medicine, Rehabilitation \& Disabilities

Journal of Plant Science: Current Research

Journal of Psychiatry, Depression \& Anxiety

Journal of Pulmonary Medicine \& Respiratory Research

Journal of Practical \& Professional Nursing

Journal of Reproductive Medicine, Gynaecology \& Obstetrics

Journal of Stem Cells Research, Development \& Therapy

Journal of Surgery: Current Trends \& Innovations

Journal of Toxicology: Current Research

Journal of Translational Science and Research

Trends in Anatomy \& Physiology

Journal of Vaccines Research \& Vaccination

Journal of Virology \& Antivirals

Submit Your Manuscript: http://www.heraldopenaccess.us/Online-Submission.php 